\title{
New method for detecting slime production by coagulase negative staphylococci
}

\author{
D J FREEMAN, F R FALKINER, C T KEANE Department of Clinical Microbiology, Sir Patrick \\ Dun's Laboratory, St James's Hospital, Dublin, Eire
}

SUMMARY An alternative method for detecting the production of slime by coagulase negative staphylococci was compared with the routinely used Christensen method on 124 isolates of coagulase negative staphylococci from carriage sites, blood cultures, and infected peritoneal dialysis fluids. The alternative method requires the use of a specially prepared solid medium - brain heart infusion broth, supplemented with $5 \%$ sucrose, and Congo red stain. Of the 124 tests, there was complete agreement between methods in 107 and only one strain was clearly negative by Christensen's method while positive on Congo red agar.

The Congo red method is rapid, sensitive, and reproducible and has the advantage that colonies remain viable on the medium. It is also not subject to interbatch variation of media which sometimes affects the reproducibility of the Christensen method.

The increased use of new invasive procedures entailing the use of prosthetic devices has had considerable impact on the role of coagulase negative staphylococci in clinical medicine. Their emergence as "opportunistic pathogens" among patients receiving particular types of treatment, such as continuous ambulatory peritoneal dialysis (CAPD), has stimulated considerable interest in their mode of pathogenesis. Since the observation by Bayston and Penny that some strains of coagulase negative staphylococci produced a mucoid exopolysaccharide,' a great deal of work has been devoted to evaluating the clinical importance of slime as a potential indicator of virulence.

There have been numerous reports of the distribution of slime production among clinically important coagulase negative staphylococci isolates. ${ }^{23}$ These studies suggest that the production of slime facilitates adherence of coagulase negative staphylococci to biopolymers ${ }^{4}$ and may be responsible for poor therapeutic response and relapse. ${ }^{5}$ Slime has also been shown to inhibit the cell mediated immune response in vitro. $^{6}$

The production of slime by coagulase negative staphylococci is routinely detected using the Christensen method. ${ }^{7}$ This method is not always successful for detecting weak slime production and variations in media may affect the result. We describe an alternative method for detecting slime that uses a specially prepared solid medium and compare it with the Christensen method.

Accepted for publication 16 February 1989

\section{Material and methods}

One hundred and twenty four isolates of coagulase negative staphylococci were obtained from carriage sites, blood cultures, and infected peritoneal dialysis fluids. Isolates were initially identified by Gram stain, bacitracin resistance, coagulase reaction and catalase test. Known slime producing and non-slime producing strains of Staphylococcus epidermidis were included as controls.

\section{CHRISTENSEN METHOD}

Strains were tested for slime production by a modification of the standard method of Christensen. ${ }^{7}$ Two millilitre volumes of tryptic soy broth (TSB, Difco Laboratories Ltd, Michigan), in $12 \times 75 \mathrm{~mm}$ borosilicate test tubes were inoculated with single colonies and incubated statically for 48 hours at $37^{\circ} \mathrm{C}$, after which the contents were decanted and $1 \mathrm{ml}$ volumes of a $0.4 \%$ aqueous solution of trypan blue (Sigma Chemical Co, St Louis, Missouri) were added. Each tube was then gently rotated to ensure uniform staining of any adherent material on the inner surface and the contents gently decanted. The tubes were then placed upside down to drain. A positive result was indicated by the presence of an adherent layer of stained material on the inner surface of the tube. Presence of stained material at the liquid-air interface alone was not regarded as indicative of slime production.

TSB is the medium of choice for the Christensen method, so we compared a selection of strains for 
slime production using the conventional Christensen method and a modified method using brain heart infusion broth (BHI, Oxoid) and found that the discrepancy between methods was no greater than that observed when different batches of TSB were used. In fact, these latter discrepancies have occasionally required us to increase the amount of dehydrated TSB powder used in medium preparation to achieve satisfactory results with the positive slime controls using the Christensen method. In our experience the use of extended incubation time ( 48 hours) for the Christensen test provides enhanced discrimination of positive and negative results, reducing the number of indeterminate results.

\section{CONGO RED AGAR METHOD}

The medium was composed of brain heart infusion broth (Oxoid Ltd, Basingstoke, England) $37 \mathrm{~g} / \mathrm{l}$, sucrose $50 \mathrm{~g} / \mathrm{l}$, agar No 1 (Oxoid) $10 \mathrm{~g} / \mathrm{l}$ and Congo red (BDH Ltd) $0.8 \mathrm{~g} / \mathrm{l}$. Congo red stain was prepared as a concentrated aqueous solution and autoclaved $\left(121^{\circ} \mathrm{C}\right.$ for 15 minutes) separately from the other medium constituents, and was then added when the agar had cooled to $55^{\circ} \mathrm{C}$. Plates of the medium were inoculated and incubated aerobically for 24 hours at $37^{\circ} \mathrm{C}$. A positive result was indicated by black colonies with a dry crystalline consistency. Non-slime producers usually remained pink, though occasional darkening at the centre of the colonies was observed and this gave a bulls eye appearance. An indeterminate result was indicated by a darkening of the colonies but with the absence of a dry crystalline colonial morphology.

\section{Results}

The results show that there was complete agreement between methods in 107 out of a total of 124 tests (table). For the remaining 17 strains, 16 discrepancies were observed, though only on the basis of a weak reaction difference and only one strain was clearly negative by Christensen's method while positive on Congo red agar. The figure shows the appearance of

Table Comparison of methods for detecting slime production

\begin{tabular}{lll}
\hline No of tests & Congo red medium & Christensen method \\
\hline 51 & + & + \\
56 & - & - \\
7 & + & \pm \\
6 & - & - \\
2 & \pm & + \\
1 & \pm & - \\
1 & + & \\
\hline
\end{tabular}

+ Positive result; \pm weak or indeterminate result; - negative result.

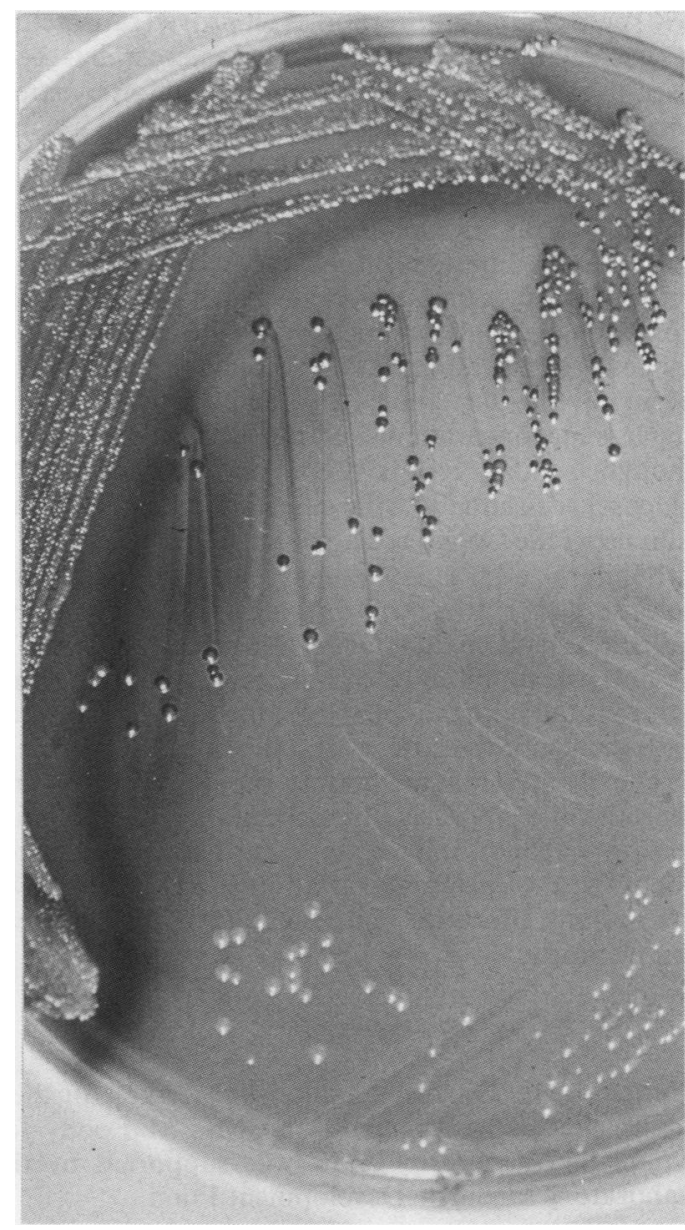

Figure Slime producing strain (top) and non-slimeproducing strain (bottom) of coagulase negative staphylococci grown on Congo red medium. Colonies of the slime producing strain show distinctive blackening.

both a slime producer and non-slime producer on the Congo red medium, respectively.

\section{Discussion}

Although capsules produced by staphylococci have been detected by changes in colonial appearance, ${ }^{89}$ this approach has not been found suitable for determining slime production. Both methods described here are based on the enhancement of exopolysaccharide production by using enriched media, TSB, in the Christensen method, ${ }^{7}$ while the Congo red agar method also requires the use of a highly nutritious medium - in this case, brain heart infusion broth with 
$5 \%$ sucrose supplementation. As it proved satisfactory it was retained thereafter. Added sucrose has been used for the detection of glucan production by streptococcal species. ${ }^{10}$ Congo red stain was chosen because it has been used as a stain for showing the presence of the exopolysaccharide of aquatic Gram negative bacilli by light microscopical examination.

The exact mechanism of the Congo red phenomenon is as yet unknown, although it is clear that the colour change only occurs towards the latter stages of incubation suggesting that a secondary product may be involved. Our studies also show that the use of sugar supplementation (sucrose was normally used though similar results were obtained using $5 \%$ glucose) is essential for the detection of slime production using the Congo red medium.

Similarly, addition of Congo red stain $(0.08 \%)$ and sucrose $(5 \%)$ to trypticase soya agar or a less nutritious medium like Direct Sensitivity Agar (DST, Oxoid) was found to be equally efficient for detecting slime production, though the colour change associated with the use of the latter was sometimes less intense.

The Congo red method is rapid, sensitive, and reproducible and has the advantage that colonies remain viable on the medium. Furthermore, it is not subject to the interbatch variation of media which, we have found, affects the reproducibility of the Christensen method.

We thank Drs D Coleman and M Cafferkey for their advice and encouragement, and Mrs M Foody for typing the manuscript. DJF was supported by the Laboratory Medicine Development Fund.

\section{References}

1 Bayston R, Penny SR. Excessive production of mucoid substance in Staphylococcus SIIA: a possible factor in colonisation of Holter shunts. Dev Med Child Neurol 1972;14(Supp 27):25-8.

2 Ishak MA, Groschel DH, Mandell GL, Wenzel RP. Association of slime with pathogenicity of coagulase negative staphylococci causing nosocomial septicemia. J Clin Microbiol 1985;22: 1025-9.

3 Younger JJ, Christensen GD, Bartley DL, Simmons JC, Barrett FF. Coagulase negative staphylococci isolated from cerebrospinal fluid shunts: importance of slime production, species identification and shunt removal to clinical outcome. J Infect Dis 1987;156:548-53.

4 Peters G, Pulverer G. Pathogenesis and management of Staphylococcus epidermidis 'plastic' foreign body infections. $J$ Antimicrob Chemother 1984;14(Suppl D):67-71.

5 Kristinsson KG, Spencer RC, Brown CB. Clinical importance of production of slime by coagulase negative staphylococci in chronic ambulatory peritoneal dialysis. J Clin Pathol 1986;39:117-18.

6 Johnson GM, Lee DA, Regelmann WE, Gray ED, Peters G, Quie PG. Interference with granulocyte function by Staphylococcus epidermidis slime. Infect Immun 1986;54:13-20.

7 Christensen GD, Simpson WA, Bisno AL, Beachey EH. Adherence of slime-producing strains of Staphylococcus epidermidis to smooth surfaces. Infect Immun 1982;37:318-26.

8 Smith RM, Parisi JT, Vidal L, Baldwin JN. Nature of the genetic determinant controlling encapsulation in Staphylococcus aureus Smith. Infect Immun 1977;17:231-4.

9 Finkelstein RA, Sulkin SE. Characteristics of coagulase-positive and coagulase-negative staphylococci in serum-soft agar. $J$ Bacteriol 1958;75:339-44.

10 Vera HD, Power DA. Sucrose broth. In: Lennette, EH, ed. Manual of clinical microbiology. Third Edition. Washington, DC: American Society of Microbiology, 1980:998.

11 Allison DG, Sutherland IW. A staining technique for attached bacteria and its correlation to extracellular carbohydrate production. J Microbiol Methods 1984;2:93-9.

Requests for reprints to: Dr D J Freeman, Department of Clinical Microbiology, Central Pathology Laboratory, St James's Hospital, James's Street, Dublin 8, Eire. 